\title{
Simultaneous iterative algorithm for the split equality fixed-point problem of demicontractive mappings
}

\author{
Yaqin Wang ${ }^{\mathrm{a}, *}$, Tae Hwa Kim ${ }^{\mathrm{b}}$ \\ ${ }^{a}$ Department of Mathematics, Shaoxing University, Shaoxing 312000, China. \\ ${ }^{b}$ Department of Applied Mathematics, College of Natural Sciences, Pukyong National University, Busan 48513, Republic of Korea. \\ Communicated by Y. H. Yao
}

\begin{abstract}
In this paper, we investigate a new simultaneous iterative algorithm for the split equality fixed-point problem of demicontractive mappings in real Hilbert spaces and obtain a strong convergence result with no compactness assumptions on the spaces or the mappings and with no extra conditions on the fixed point sets. The results obtained in this paper generalize and improve the recent ones announced by many others. (c)2017 All rights reserved.
\end{abstract}

Keywords: Split equality fixed-point problem, demicontractive mapping, strong convergence, iterative algorithm. 2010 MSC: 47H09, 47H10, 47J05, 49J53.

\section{Introduction}

The split feasibility problem (SFP) arises in many areas of applications such as phase retrieval, medical image reconstruction, image restoration and radiation therapy treatment planning (see, e.g. Byrne [1], Censor et al. [2] and Censor and Elfving [3]). It is formulated as follows:

$$
\text { Find } x^{*} \in C \text { such that } A x^{*} \in Q \text {, }
$$

where $C$ and $Q$ are respectively closed convex subsets in Hilbert spaces $H_{1}$ and $H_{2}$, and $A: H_{1} \rightarrow H_{2}$ is a bounded linear mapping. The SFP was first introduced in 1994 by Censor and Elfving [3] in finitedimensional Hilbert spaces for modelling inverse problems arising from phase retrieval and medical image reconstruction. Recently the SFP has been widely studied by many authors (see, e.g., [15-17]). An efficient algorithm for solving the SFP is Byrne's CQ algorithm [1]: for any $x_{0} \in \mathrm{H}_{1}$, the CQ algorithm generates an iterative sequence as

$$
x_{k+1}=P_{C}\left(I+\gamma A^{*}\left(P_{Q}-I\right) A\right) x_{k}
$$

where $0<\gamma<2 /\|A\|^{2}, P_{C}$ and $P_{Q}$ are the metric projections from $H_{1}$ onto $C$ and from $\mathrm{H}_{2}$ onto $Q$, respectively. It is known that the CQ algorithm converges weakly to a solution of the SFP (1.1), if such a solution exists.

\footnotetext{
*Corresponding author

Email addresses: wangyaqin0579@126.com (Yaqin Wang), taehwa1225@gmail.com (Tae Hwa Kim)
} 
In 2009, Censor and Segal [4] introduced the following split common fixed-point problem (SCFP):

Find $x \in F(U)$ such that $A x \in F(T)$,

where $A: \mathrm{H}_{1} \rightarrow \mathrm{H}_{2}$ is a bounded linear operator, $\mathrm{U}: \mathrm{H}_{1} \rightarrow \mathrm{H}_{1}$ and $\mathrm{T}: \mathrm{H}_{2} \rightarrow \mathrm{H}_{2}$ are two nonexpansive operators, $\mathrm{F}(\mathrm{U})=\mathrm{C}$ and $\mathrm{F}(\mathrm{T})=\mathrm{Q}$ are the fixed point sets of $\mathrm{U}$ and $\mathrm{T}$, respectively.

To solve the SCFP (1.2), Censor and Segal [4] proposed and proved, in finite-dimensional spaces, the convergence of the following algorithm

$$
x_{k+1}=U\left(x_{k}+\gamma A^{t}(T-I) A x_{k}\right), \quad k \in N,
$$

where $\gamma \in\left(0, \frac{2}{\lambda}\right)$ with $\lambda$ being the largest eigenvalue of the matrix $A^{t} A$ ( $A^{t}$ stands for matrix transposition).

Let $\mathrm{H}_{1}, \mathrm{H}_{2}, \mathrm{H}_{3}$ be real Hilbert spaces. In 2013, Moudafi [9] proposed a new split equality problem (SEP): Let $A: \mathrm{H}_{1} \rightarrow \mathrm{H}_{3}, \mathrm{~B}: \mathrm{H}_{2} \rightarrow \mathrm{H}_{3}$ be two bounded linear operators, $\mathrm{C} \subset \mathrm{H}_{1}, \mathrm{Q} \subset \mathrm{H}_{2}$ be two nonempty closed convex sets.

$$
\text { Find } x \in C, y \in Q \text { such that } A x=B y .
$$

In addition, assume that $\mathrm{U}: \mathrm{H}_{1} \rightarrow \mathrm{H}_{1}$ and $\mathrm{T}: \mathrm{H}_{2} \rightarrow \mathrm{H}_{2}$ are two firmly quasi-nonexpansive operators, Moudafi [10] introduced the following split equality fixed-point problem (SEFP):

$$
\text { Find } x \in C:=F(U), y \in Q:=F(T) \text { such that } A x=B y \text {, }
$$

which allows asymmetric and partial relations between the variables $x$ and $y$. The interest is to cover many situations, for instance, in decomposition methods for PDF's, applications in game theory and in intensity-modulated radiation therapy (IMRT). Note that the SEFP (1.4) reduces to the SCFP (1.2) if $\mathrm{H}_{2}=\mathrm{H}_{3}$ and $\mathrm{B}=\mathrm{I}$ (where $\mathrm{I}$ is the identity operator on $\mathrm{H}_{2}$ ) in (1.4).

For solving the SEFP (1.4), Moudafi [10] introduced the following iterative scheme:

$$
\left\{\begin{array}{l}
x_{k+1}=U\left(x_{k}-\gamma_{k} A^{*}\left(A x_{k}-B y_{k}\right)\right) \\
y_{k+1}=T\left(y_{k}+\gamma_{k} B^{*}\left(A x_{k+1}-B y_{k}\right)\right)
\end{array}\right.
$$

where $\gamma_{k} \in\left(\epsilon, \min \left\{\frac{1}{\lambda_{A}}, \frac{1}{\lambda_{B}}\right\}-\epsilon\right), \lambda_{A}$ and $\lambda_{B}$ are the spectral radius of $A^{*} A$ and $B^{*} B$, respectively. Using this iterative scheme, he obtained a weak convergence for the SEFP (1.4).

Subsequently, Moudafi and Al-Shemas [11] introduced the following simultaneous iterative method to solve the SEFP (1.4):

$$
\left\{\begin{array}{l}
x_{k+1}=U\left(x_{k}-\gamma_{k} A^{*}\left(A x_{k}-B y_{k}\right)\right) \\
y_{k+1}=T\left(y_{k}+\gamma_{k} B^{*}\left(A x_{k}-B y_{k}\right)\right)
\end{array}\right.
$$

for firmly quasi-nonexpansive operators $U$ and $T$, where $\gamma_{k} \in\left(\epsilon, \frac{2}{\lambda_{A}+\lambda_{B}}-\epsilon\right), \lambda_{A}$ and $\lambda_{B}$ are the spectral radius of $A^{*} A$ and $B^{*} B$, respectively. Using the iterative scheme (1.5), they obtained a weak convergence for the SEFP (1.4).

Recently, Zhao and He [18] introduced the following simultaneous iterative algorithm for solving the SEFP (1.4): For any $x_{0} \in \mathrm{H}_{1}, \mathrm{y}_{0} \in \mathrm{H}_{2}$,

$$
\left\{\begin{array}{l}
u_{k}=x_{k}-\gamma_{k} A^{*}\left(A x_{k}-B y_{k}\right), \\
x_{k+1}=\alpha_{k} u_{k}+\left(1-\alpha_{k}\right) u\left(u_{k}\right), \\
v_{k}=y_{k}+\gamma_{k} B^{*}\left(A x_{k}-B y_{k}\right), \\
y_{k+1}=\beta_{k} v_{k}+\left(1-\beta_{k}\right) T\left(v_{k}\right),
\end{array}\right.
$$

where $\gamma_{\mathrm{k}}$ is the same as in (1.5), $\mathrm{A}: \mathrm{H}_{1} \rightarrow \mathrm{H}_{3}, \mathrm{~B}: \mathrm{H}_{2} \rightarrow \mathrm{H}_{3}$ are two bounded linear operators, $\mathrm{U}: \mathrm{H}_{1} \rightarrow \mathrm{H}_{1}$ and $\mathrm{T}: \mathrm{H}_{2} \rightarrow \mathrm{H}_{2}$ are two quasi-nonexpansive operators. They proved the weak convergence of this algorithm. 
Very recently, Shehu and Ogbuisi [12] used a modified Mann iterative algorithm

$$
\left\{\begin{array}{l}
x_{0} \in H_{1}, \\
w_{k}=\left(1-\alpha_{k}\right) x_{k}, \\
y_{k}=w_{k}+\gamma A^{*}(T-I) A w_{k}, \\
x_{k+1}=\left(1-\beta_{k}\right) y_{k}+\beta_{k} U y_{k},
\end{array}\right.
$$

to approximate the solution of the SCFP (1.2) for demicontractive mappings in a real Hilbert space and obtained a strong convergence result with no compactness assumptions.

Motivated by the above works, in this paper, we introduce a new simultaneous iterative scheme for solving the SEFP (1.4):

$$
\left\{\begin{array}{l}
\forall x_{0} \in H_{1}, y_{0} \in H_{2} \\
u_{k}=x_{k}-\gamma_{k} A^{*}\left(A x_{k}-B y_{k}\right) \\
w_{k}=\left(1-t_{k}\right) u_{k} \\
x_{k+1}=\alpha_{k} w_{k}+\left(1-\alpha_{k}\right) U\left(w_{k}\right) \\
v_{k}=y_{k}+\gamma_{k} B^{*}\left(A x_{k}-B y_{k}\right) \\
p_{k}=\left(1-t_{k}\right) v_{k} \\
y_{k+1}=\beta_{k} p_{k}+\left(1-\beta_{k}\right) T\left(p_{k}\right)
\end{array}\right.
$$

and obtain a strong convergence result with no compactness assumptions on the spaces or the mappings and with no extra conditions on the fixed point sets.

\section{Preliminaries}

Throughout this paper, let $\mathbb{N}$ and $\mathbb{R}$ be the set of positive integers and real numbers, respectively. Let $\mathrm{H}$ be a real Hilbert space with inner product $\langle\cdot, \cdot\rangle$ and norm $\|\cdot\|$. Let $\mathrm{C}$ be a nonempty closed convex subset of $\mathrm{H}$ and $\mathrm{T}$ be a mapping of $\mathrm{C}$ into $\mathrm{H}$. We denote the set of fixed points of $T$ by $F(T)$. We always denote strong and weak convergence by " $\rightarrow$ and $\rightarrow$ ", respectively, and use $\omega_{w}\left(x_{k}\right)=\left\{x: \exists x_{k_{j}} \rightarrow x\right\}$ to denote the weak $\omega$-limit set of $\left\{x_{k}\right\}$.

Definition 2.1. Let $H$ be a real Hilbert space. An operator $T: H \rightarrow H$ is said to be

(i) nonexpansive, if $\|\mathrm{T} x-\mathrm{T} y\| \leqslant\|x-y\|, \forall x, y \in H$;

(ii) quasi-nonexpansive, if $F(T) \neq \emptyset$ and if $\|T x-q\| \leqslant\|x-q\|$ for all $x \in H$ and $q \in F(T)$;

(iii) firmly nonexpansive, if

$$
\|T x-T y\|^{2} \leqslant\|x-y\|^{2}-\|(I-T) x-(I-T) y\|^{2}, \quad \forall x, y \in H,
$$

or equivalently,

$$
\|\mathrm{T} x-\mathrm{T} y\|^{2} \leqslant\langle x-y, \mathrm{~T} x-\mathrm{T} y\rangle, \quad \forall x, y \in \mathrm{H} ;
$$

(iv) firmly quasi-nonexpansive, if $F(T) \neq \emptyset$ and

$$
\|\mathrm{T} x-\mathrm{q}\|^{2} \leqslant\|x-\mathrm{q}\|^{2}-\|x-\mathrm{T} x\|^{2}, \quad \forall x \in H, \quad \mathrm{q} \in \mathrm{F}(\mathrm{T}) ;
$$

(v) $\mu$-demicontractive, if $\mathrm{F}(\mathrm{T}) \neq \emptyset$ and there exists a constant $\mu \in(-\infty, 1)$ such that

$$
\|\mathrm{T} x-\mathrm{q}\|^{2} \leqslant\|x-\mathrm{q}\|^{2}+\mu\|x-\mathrm{T} x\|^{2}, \quad \forall x \in H, \quad \mathrm{q} \in \mathrm{F}(\mathrm{T}) .
$$

In Hilbert spaces, (2.1) is equivalent to

$$
2\langle T x-x, x-q\rangle \leqslant(\mu-1)\|x-T x\|^{2} .
$$

Remark 2.2. Notice that 0-demicontractive is exactly quasi-nonexpansive. In particular, we say that it is quasi-strict pseudo-contractive [7], if $0 \leqslant \mu<1$. Moreover, if $\mu \leqslant 0$, every $\mu$-demicontractive mapping 
becomes quasi-nonexpansive. Therefore, it is sufficient only to take $\mu \in(0,1)$ in (v) of Definition 2.1, or as the notion of quasi-strict pseudo-contraction due to [7].

Remark 2.3. The following inclusions are obvious.

Firmly quasi-nonexpansive mappings $\subset$ quasi-nonexpansive mappings $\subset$ demicontractive mappings.

Example 2.4. Let $\mathrm{H}=\ell_{2}$ and $\mathrm{T}: \ell_{2} \rightarrow \ell_{2}$ be defined by $\mathrm{T}\left(x_{1}, x_{2}, x_{3}, \cdots\right)=\left(0, x_{1}, x_{2}, x_{3}, \cdots\right)$, for arbitrary $\left(x_{1}, x_{2}, x_{3}, \cdots\right) \in \ell_{2}$. Then $T$ is a quasi-nonexpansive mapping which is not firmly quasi-nonexpansive mapping. have

In fact, it is obvious that $T$ has a unique fixed point $x=0$. For arbitrary $x=\left(x_{1}, x_{2}, x_{3}, \cdots\right) \in \ell_{2}$, we

$$
\|\mathrm{T} x-0\|=\|x-0\|,
$$

so $T$ is quasi-nonexpansive. For every $x \neq 0$, suppose

$$
\|T x-0\|^{2} \leqslant\|x-0\|^{2}-\|x-T x\|^{2},
$$

which implies that $x=T x$. Thus $x=0$, which is a contradiction. Therefore, $T$ is not firmly quasinonexpansive.

Example 2.5. Let $\mathrm{H}=\ell_{2}$ and $\mathrm{T}: \ell_{2} \rightarrow \ell_{2}$ be defined by $\mathrm{T} x=-k x$, for arbitrary $x \in \ell_{2}$, where $k>1$. Then $\mathrm{F}(\mathrm{T})=\{0\}$ and $\mathrm{T}$ is a demicontractive mapping which is not quasi-nonexpansive.

In fact, it is obvious that $T$ has a unique fixed point $x=0$. For each $x \in \ell_{2}$, we have

$$
\|\mathrm{T} x-0\|^{2}=k^{2}\|x-0\|^{2},
$$

which implies that $\mathrm{T}$ is not quasi-nonexpansive. And

$$
\|x-T x\|^{2}=\|x-(-k x)\|^{2}=(1+k)^{2}\|x-0\|^{2},
$$

which implies that

$$
\|x-0\|^{2}=\frac{1}{(1+k)^{2}}\|x-T x\|^{2} .
$$

Thus we have

$$
\|\mathrm{T} x-0\|^{2}=\|x-0\|^{2}+\left(k^{2}-1\right)\|x-0\|^{2}=\|x-0\|^{2}+\frac{k^{2}-1}{(k+1)^{2}}\|x-T x\|^{2} .
$$

It follows from $\frac{k^{2}-1}{(k+1)^{2}} \in(0,1)$ that $T$ is a $\frac{k^{2}-1}{(k+1)^{2}}$-demicontractive mapping.

In real Hilbert spaces, we easily get the following equality:

$$
2\langle x, y\rangle=\|x\|^{2}+\|y\|^{2}-\|x-y\|^{2}, \forall x, y \in H .
$$

The metric (or nearest point) projection $\mathrm{P}_{C}$ from $\mathrm{H}$ onto $\mathrm{C}$ is defined as follows: Given $x \in H$, the unique point $\mathrm{P}_{\mathrm{C}} x \in \mathrm{C}$ satisfies the property

$$
\left\|x-P_{C} x\right\|=\inf _{y \in C}\|x-y\| .
$$

It is well-known [13] that $\mathrm{P}_{\mathrm{C}}$ is a nonexpansive mapping and is characterized by the inequality

$$
P_{C} x \in C,\left\langle x-P_{C} x, y-P_{C} x\right\rangle \leqslant 0, \forall y \in C .
$$

Definition 2.6. Let $T: H \rightarrow H$ be a nonlinear mapping. Then $T$ is said to be demiclosed at $y \in H$, if $x_{n} \rightarrow x \in H$ and $T x_{n} \rightarrow y$, then $y=T x$. 
In what follows, we shall make use of the following lemmas.

Lemma 2.7 ([14]). Assume that $\left\{a_{k}\right\}$ is a sequence of nonnegative real numbers such that

$$
a_{k+1} \leqslant\left(1-\lambda_{k}\right) a_{k}+\lambda_{k} \delta_{k}, k \geqslant 0,
$$

where $\left\{\lambda_{k}\right\}$ is a sequence in $(0,1)$ and $\left\{\delta_{k}\right\}$ is a sequence in $\mathbb{R}$ such that

(a) $\sum_{k=0}^{\infty} \lambda_{k}=\infty$ and $\lim _{k \rightarrow \infty} \lambda_{k}=0$;

(b) $\lim \sup _{k \rightarrow \infty} \delta_{k} \leqslant 0$ or $\sum_{k=0}^{\infty} \lambda_{k}\left|\delta_{k}\right|<\infty$.

Then $\lim _{k \rightarrow \infty} a_{k}=0$.

Lemma 2.8 ([7, Proposition 2.1 (iii)]). Assume $\mathrm{C}$ is a closed convex subset of a Hilbert space $\mathrm{H}$. Let $\mathrm{T}: \mathrm{C} \rightarrow \mathrm{C}$ be a self-mapping of $\mathrm{C}$. If $\mathrm{T}$ is a $\mu$-demicontractive mapping (which is also called $\mu$-quasi-strictly-contraction in [7]), then the fixed point set $\mathrm{F}(\mathrm{T})$ is closed and convex.

Lemma 2.9 ([6]). Let $\left\{\Gamma_{n}\right\}$ be a sequence of real numbers that does not decrease at infinity in the sense that there exists a subsequence $\left\{\Gamma_{n_{i}}\right\}$ of $\left\{\Gamma_{n}\right\}$ which satisfies $\Gamma_{n_{i}}<\Gamma_{n_{i}+1}$, for all $i \in \mathbb{N}$. Define the sequence $\{\tau(n)\}_{n} \geqslant n_{0}$ of integers as follows:

$$
\tau(\mathrm{n})=\max \left\{\mathrm{k} \leqslant \mathrm{n}: \Gamma_{\mathrm{k}}<\Gamma_{\mathrm{k}+1}\right\},
$$

where $\mathrm{n}_{0} \in \mathbb{N}$ such that $\left.\left\{\mathrm{k} \leqslant \mathrm{n}_{0}: \Gamma_{\mathrm{k}}<\Gamma_{\mathrm{k}+1}\right\}\right\} \neq \emptyset$. Then, the following hold:

(i) $\tau\left(n_{0}\right) \leqslant \tau\left(n_{0}+1\right) \leqslant \cdots$, and $\tau(n) \rightarrow \infty$;

(ii) $\Gamma_{\tau(n)} \leqslant \Gamma_{\tau(n)+1}$ and $\Gamma_{\mathrm{n}} \leqslant \Gamma_{\tau(\mathrm{n})+1}, \forall \mathrm{n} \geqslant \mathrm{n}_{0}$.

Lemma 2.10 ([5], demiclosedness principle). Let $\mathrm{H}$ be a real Hilbert space, $\mathrm{C}$ a nonempty closed convex subset of $\mathrm{H}$, and $\mathrm{T}: \mathrm{C} \rightarrow \mathrm{H}$ a nonexpansive mapping. Then the mapping $\mathrm{I}-\mathrm{T}$ is demiclosed on $\mathrm{C}$, where $\mathrm{I}$ is the identity mapping; that is, $\mathrm{x}_{\mathrm{n}} \rightarrow \mathrm{x}$ in $\mathrm{H}$ and $(\mathrm{I}-\mathrm{T}) \mathrm{x}_{\mathrm{n}} \rightarrow \mathrm{y}$ imply that $\mathrm{x} \in \mathrm{C}$ and $(\mathrm{I}-\mathrm{T}) \mathrm{x}=\mathrm{y}$.

Here the following question is naturally raised: If $\mathrm{T}: \mathrm{C} \rightarrow \mathrm{H}$ is quasi-nonexpansive, is $\mathrm{I}-\mathrm{T}$ still demiclosed at 0 ? The answer is negative as follows.

Example 2.11. The mapping $T:[0,1] \rightarrow[0,1]$ is defined by

$$
T x=\left\{\begin{array}{l}
\frac{x}{5}, \quad x \in\left[0, \frac{1}{2}\right] \\
x \sin \pi x, \quad x \in\left(\frac{1}{2}, 1\right] .
\end{array}\right.
$$

Then $\mathrm{T}$ is a quasi-nonexpansive mapping, but $\mathrm{I}-\mathrm{T}$ is not demiclosed at 0 .

In fact, $F(T)=\{0\}$. For any $x \in\left[0, \frac{1}{2}\right]$, we have

$$
|\mathrm{T} x-0|=\left|\frac{x}{5}-0\right| \leqslant|x-0|,
$$

and for any $x \in\left(\frac{1}{2}, 1\right]$, we have

$$
|\mathrm{T} x-0|=|x \sin \pi x-0| \leqslant|x-0| .
$$

Thus $T$ is quasi-nonexpansive. By taking $\left\{x_{n}\right\} \subset\left(\frac{1}{2}, 1\right]$ and $x_{n} \rightarrow \frac{1}{2}(n \rightarrow \infty)$, we have

$$
\left|(I-T) x_{n}\right|=\left|x_{n}\left[1-\sin \pi x_{n}\right]\right| \rightarrow 0(n \rightarrow \infty) .
$$

But $\mathrm{T} \frac{1}{2}=\frac{1}{10} \neq \frac{1}{2}$, i.e., $(\mathrm{I}-\mathrm{T}) \frac{1}{2} \neq 0$, so $\mathrm{I}-\mathrm{T}$ is not demiclosed at 0 .

Remark 2.12. Notice that a demicontractive mapping could enjoy the demiclosedness property at the origin, for an example, let $\mathrm{C}$ be the unit ball of $\mathrm{H}=\ell_{2}$ and let $\mathrm{T}: \mathrm{C} \rightarrow \mathrm{H}$ be defined as in Example 2.5. Then $T$ is not quasi-nonexpansive but $\mu$-demicontactive, where $\mu:=\frac{k^{2}-1}{(k+1)^{2}}$. However, $I-T$ is obviously demiclosed at the origin. For, whenever $\left\{x_{n}\right\}$ is any sequence in $C$ such that $x_{n} \rightarrow x \in C$ and $\left\|x_{n}-T x_{n}\right\| \rightarrow 0$, we readily see that $x=0 \in F(T)$. 


\section{Main results}

Throughout this section, we assume that $\mathrm{H}_{1}, \mathrm{H}_{2}, \mathrm{H}_{3}$ are three real Hilbert spaces. Put $\mathrm{H}^{*}=\mathrm{H}_{1} \times \mathrm{H}_{2}$. Define the inner product of $\mathrm{H}^{*}$ as follows:

$$
\left\langle\left(x_{1}, y_{1}\right),\left(x_{2}, y_{2}\right)\right\rangle=\left\langle x_{1}, x_{2}\right\rangle+\left\langle y_{1}, y_{2}\right\rangle, \quad \forall\left(x_{1}, y_{1}\right),\left(x_{2}, y_{2}\right) \in H^{*} .
$$

It is easy to see that $\mathrm{H}^{*}$ is also a real Hilbert space and

$$
\|(x, y)\|=\left(\|x\|^{2}+\|y\|^{2}\right)^{\frac{1}{2}}, \quad \forall(x, y) \in H^{*} .
$$

Lemma 3.1. Given two bounded linear operators $A: \mathrm{H}_{1} \rightarrow \mathrm{H}_{3}$, B : $\mathrm{H}_{2} \rightarrow \mathrm{H}_{3}$, let $\mathrm{U}: \mathrm{H}_{1} \rightarrow \mathrm{H}_{1}$ and $\mathrm{T}: \mathrm{H}_{2} \rightarrow \mathrm{H}_{2}$ be $\mu$-demicontractive and $v$-demicontractive, respectively. Assume that the solution set $\Omega$ of (1.4) is nonempty, i.e.,

$$
\Omega:=\{(x, y) \mid x \in F(U), y \in F(T) \text { such that } \mathrm{Ax}=\mathrm{B} y\} \neq \emptyset .
$$

Then $\Omega$ is a nonempty closed convex set.

Proof. By Lemma 2.8 we have $\mathrm{F}(\mathrm{T})$ and $\mathrm{F}(\mathrm{U})$ are both closed convex subsets, and since $\mathrm{A}$ and $\mathrm{B}$ are both linear, it is easy to see that $\Omega$ is a closed convex subset in $\mathrm{H}^{*}$.

Let $P_{\Omega}$ be a metric projection from $H^{*}$ onto $\Omega$. We denote the origins of $H_{1}$ and $H_{2}$ by $\theta_{1}$ and $\theta_{2}$, respectively.

Theorem 3.2. Let the mappings $\mathrm{A}, \mathrm{B}, \mathrm{U}, \mathrm{T}$ be the same as in Lemma 3.1. Assume that $\mathrm{U}-\mathrm{I}$ and $\mathrm{T}-\mathrm{I}$ are demiclosed at the orgins and the solution set $\Omega$ of (1.4) is nonempty. Let $\gamma_{k} \in\left(\epsilon, \frac{2}{\lambda_{A}+\lambda_{B}}-\epsilon\right), \lambda_{A}$ and $\lambda_{B}$ be the spectral radius of $A^{*} A$ and $B^{*} B$ respectively and $\epsilon$ is small enough. Suppose $\left\{t_{k}\right\}$ is a sequence in $(0,1)$ satisfying $\lim _{k \rightarrow \infty} t_{k}=0$ and $\sum_{k=0}^{\infty} t_{k}=\infty$. Then the sequence $\left\{\left(x_{k}, y_{k}\right)\right\}$ generalized by (1.6) strongly converges to $a$ solution $\mathrm{P}_{\Omega}\left(\theta_{1}, \theta_{2}\right)$ of $(1.4)$, provided that $\left\{\alpha_{k}\right\} \subset(\mu+\delta, 1-\delta)$ and $\left\{\beta_{k}\right\} \subset(\nu+\sigma, 1-\sigma)$ for small enough $\delta, \sigma>0$.

Proof. Set $\left(x^{*}, y^{*}\right)=P_{\Omega}\left(\theta_{1}, \theta_{2}\right)$. By (2.3) we have

$$
\left\langle\left(\theta_{1}, \theta_{2}\right)-\left(x^{*}, y^{*}\right),(x, y)-\left(x^{*}, y^{*}\right)\right\rangle \leqslant 0, \quad \forall(x, y) \in \Omega,
$$

i.e.,

$$
\left\langle\left(x^{*}, y^{*}\right),\left(x-x^{*}, y-y^{*}\right)\right\rangle=\left\langle x^{*}, x-x^{*}\right\rangle+\left\langle y^{*}, y-y^{*}\right\rangle \geqslant 0, \quad \forall(x, y) \in \Omega .
$$

Since $\left(x^{*}, y^{*}\right) \in \Omega$, we have $x^{*} \in F(U), y^{*} \in F(T)$ such that $A x^{*}=B y^{*}$. It follows from the definition of $\lambda_{\mathrm{A}}$ that

$$
\begin{aligned}
\left\|A^{*}\left(A x_{k}-B y_{k}\right)\right\|^{2} & =\left\langle A^{*}\left(A x_{k}-B y_{k}\right), A^{*}\left(A x_{k}-B y_{k}\right)\right\rangle \\
& \leqslant\left\langle A x_{k}-B y_{k}, A A^{*}\left(A x_{k}-B y_{k}\right)\right\rangle \\
& \leqslant \lambda_{A}\left\|A x_{k}-B y_{k}\right\|^{2} .
\end{aligned}
$$

Similarly, we have

$$
\begin{aligned}
\left\|B^{*}\left(A x_{k}-B y_{k}\right)\right\|^{2} & =\left\langle B^{*}\left(A x_{k}-B y_{k}\right), B^{*}\left(A x_{k}-B y_{k}\right)\right\rangle \\
& \leqslant\left\langle A x_{k}-B y_{k}, B B^{*}\left(A x_{k}-B y_{k}\right)\right\rangle \\
& \leqslant \lambda_{B}\left\|A x_{k}-B y_{k}\right\|^{2} .
\end{aligned}
$$

Then by (1.6) we obtain

$$
\begin{aligned}
\left\|u_{k}-x^{*}\right\|^{2} & =\left\|x_{k}-\gamma_{k} A^{*}\left(A x_{k}-B y_{k}\right)-x^{*}\right\|^{2} \\
& =\left\|x_{k}-x^{*}\right\|^{2}-2 \gamma_{k}\left\langle x_{k}-x^{*}, A^{*}\left(A x_{k}-B y_{k}\right)\right\rangle+\gamma_{k}^{2}\left\|A^{*}\left(A x_{k}-B y_{k}\right)\right\|^{2} \\
& =\left\|x_{k}-x^{*}\right\|^{2}-2 \gamma_{k}\left\langle A x_{k}-A x^{*}, A x_{k}-B y_{k}\right\rangle+\gamma_{k}^{2}\left\|A^{*}\left(A x_{k}-B y_{k}\right)\right\|^{2} \\
& \leqslant\left\|x_{k}-x^{*}\right\|^{2}-2 \gamma_{k}\left\langle A x_{k}-A x^{*}, A x_{k}-B y_{k}\right\rangle+\gamma_{k}^{2} \lambda_{A}\left\|A x_{k}-B y_{k}\right\|^{2} .
\end{aligned}
$$


Similarly, we have

$$
\begin{aligned}
\left\|v_{k}-y^{*}\right\|^{2} & =\left\|y_{k}+\gamma_{k} B^{*}\left(A x_{k}-B y_{k}\right)-y^{*}\right\|^{2} \\
& =\left\|y_{k}-y^{*}\right\|^{2}+2 \gamma_{k}\left\langle y_{k}-y^{*}, B^{*}\left(A x_{k}-B y_{k}\right)\right\rangle+\gamma_{k}^{2}\left\|B^{*}\left(A x_{k}-B y_{k}\right)\right\|^{2} \\
& =\left\|y_{k}-y^{*}\right\|^{2}+2 \gamma_{k}\left\langle B y_{k}-B y^{*}, A x_{k}-B y_{k}\right\rangle+\gamma_{k}^{2}\left\|B^{*}\left(A x_{k}-B y_{k}\right)\right\|^{2} \\
& \leqslant\left\|y_{k}-y^{*}\right\|^{2}+2 \gamma_{k}\left\langle B y_{k}-B y^{*}, A x_{k}-B y_{k}\right\rangle+\gamma_{k}^{2} \lambda_{B}\left\|A x_{k}-B y_{k}\right\|^{2} .
\end{aligned}
$$

It follows from the above two inequalities and $A x^{*}=B y^{*}$ that

$$
\begin{aligned}
\left\|u_{k}-x^{*}\right\|^{2}+\left\|v_{k}-y^{*}\right\|^{2}= & \left\|x_{k}-x^{*}\right\|^{2}+\left\|y_{k}-y^{*}\right\|^{2}-2 \gamma_{k}\left\langle A x_{k}-A x^{*}-B y_{k}+B y^{*}, A x_{k}-B y_{k}\right\rangle \\
& +\gamma_{k}^{2}\left(\lambda_{A}+\lambda_{B}\right)\left\|A x_{k}-B y_{k}\right\|^{2} \\
\leqslant & \left\|x_{k}-x^{*}\right\|^{2}+\left\|y_{k}-y^{*}\right\|^{2}-\gamma_{k}\left[2-\gamma_{k}\left(\lambda_{A}+\lambda_{B}\right)\right]\left\|A x_{k}-B y_{k}\right\|^{2} \\
\leqslant & \left\|x_{k}-x^{*}\right\|^{2}+\left\|y_{k}-y^{*}\right\|^{2} .
\end{aligned}
$$

By (1.6), (2.1), (2.2) and the condition on $\left\{\alpha_{k}\right\}$, we have

$$
\begin{aligned}
& \left\|x_{k+1}-x^{*}\right\|^{2}=\left\|\alpha_{k}\left(w_{k}-x^{*}\right)+\left(1-\alpha_{k}\right)\left(\mathrm{U}\left(w_{k}\right)-x^{*}\right)\right\| \\
& =\alpha_{\mathrm{k}}^{2}\left\|w_{\mathrm{k}}-x^{*}\right\|^{2}+\left(1-\alpha_{\mathrm{k}}\right)^{2}\left\|\mathrm{U}\left(w_{\mathrm{k}}\right)-x^{*}\right\|^{2}+2 \alpha_{\mathrm{k}}\left(1-\alpha_{\mathrm{k}}\right)\left\langle\mathrm{U}\left(w_{\mathrm{k}}\right)-\chi^{*}, w_{\mathrm{k}}-\chi^{*}\right\rangle \\
& \leqslant \alpha_{\mathrm{k}}^{2}\left\|w_{\mathrm{k}}-x^{*}\right\|^{2}+\left(1-\alpha_{\mathrm{k}}\right)^{2}\left(\left\|w_{\mathrm{k}}-\chi^{*}\right\|^{2}+\mu\left\|\mathrm{U}\left(w_{\mathrm{k}}\right)-w_{\mathrm{k}}\right\|^{2}\right) \\
& +2 \alpha_{\mathrm{k}}\left(1-\alpha_{\mathrm{k}}\right)\left\langle\mathrm{U}\left(w_{\mathrm{k}}\right)-w_{\mathrm{k}}, w_{\mathrm{k}}-x^{*}\right\rangle+2 \alpha_{\mathrm{k}}\left(1-\alpha_{\mathrm{k}}\right)\left\langle w_{\mathrm{k}}-x^{*}, w_{\mathrm{k}}-x^{*}\right\rangle \\
& \leqslant \alpha_{\mathrm{k}}^{2}\left\|w_{\mathrm{k}}-x^{*}\right\|^{2}+\left(1-\alpha_{\mathrm{k}}\right)^{2}\left(\left\|w_{\mathrm{k}}-x^{*}\right\|^{2}+\mu\left\|\mathrm{U}\left(w_{\mathrm{k}}\right)-w_{\mathrm{k}}\right\|^{2}\right) \\
& +\alpha_{k}\left(1-\alpha_{k}\right)(\mu-1)\left\|\mathrm{U}\left(w_{k}\right)-w_{k}\right\|^{2}+2 \alpha_{k}\left(1-\alpha_{k}\right)\left\|w_{k}-x^{*}\right\|^{2} \\
& =\left\|w_{k}-x^{*}\right\|^{2}+\left(1-\alpha_{k}\right)\left(\mu-\alpha_{k}\right)\left\|\mathrm{U}\left(w_{k}\right)-w_{k}\right\|^{2} \\
& \leqslant\left\|w_{k}-x^{*}\right\|^{2} \\
& =\left\|\left(1-t_{k}\right)\left(u_{k}-x^{*}\right)+t_{k}\left(-x^{*}\right)\right\|^{2} \\
& \leqslant\left(1-t_{k}\right)\left\|u_{k}-x^{*}\right\|^{2}+t_{k}\left\|x^{*}\right\|^{2} \text {. }
\end{aligned}
$$

Similarly, we have

$$
\begin{aligned}
\left\|y_{k+1}-y^{*}\right\|^{2} & \leqslant\left\|p_{k}-y^{*}\right\|^{2}+\left(1-\beta_{k}\right)\left(v-\beta_{k}\right)\left\|T\left(p_{k}\right)-p_{k}\right\|^{2} \\
& \leqslant\left\|p_{k}-y^{*}\right\|^{2} \\
& \leqslant\left(1-t_{k}\right)\left\|v_{k}-y^{*}\right\|^{2}+t_{k}\left\|y^{*}\right\|^{2} .
\end{aligned}
$$

It follows from (3.3), (3.6) and (3.9) that

$$
\begin{aligned}
\left\|x_{k+1}-x^{*}\right\|^{2}+\left\|y_{k+1}-y^{*}\right\|^{2} & \leqslant\left(1-t_{k}\right)\left(\left\|u_{k}-x^{*}\right\|^{2}+\left\|v_{k}-y^{*}\right\|^{2}\right)+t_{k}\left(\left\|x^{*}\right\|^{2}+\left\|y^{*}\right\|^{2}\right) \\
& \leqslant\left(1-t_{k}\right)\left(\left\|x_{k}-x^{*}\right\|^{2}+\left\|y_{k}-y^{*}\right\|^{2}\right)+t_{k}\left(\left\|x^{*}\right\|^{2}+\left\|y^{*}\right\|^{2}\right) .
\end{aligned}
$$

Now, by setting $\rho_{k}\left(x^{*}, y^{*}\right)=\left\|x_{k}-x^{*}\right\|^{2}+\left\|y_{k}-y^{*}\right\|^{2}$, from the above inequality we obtain

$$
\begin{aligned}
\rho_{k+1}\left(x^{*}, y^{*}\right) & \leqslant\left(1-t_{k}\right) \rho_{k}\left(x^{*}, y^{*}\right)+t_{k}\left(\left\|x^{*}\right\|^{2}+\left\|y^{*}\right\|^{2}\right) \\
& \leqslant \max \left\{\rho_{k}\left(x^{*}, y^{*}\right),\left\|x^{*}\right\|^{2}+\left\|y^{*}\right\|^{2}\right\} .
\end{aligned}
$$

By induction, we have

$$
\rho_{\mathrm{k}+1}\left(x^{*}, \mathrm{y}^{*}\right) \leqslant \max \left\{\rho_{0}\left(x^{*}, \mathrm{y}^{*}\right),\left\|x^{*}\right\|^{2}+\left\|\mathrm{y}^{*}\right\|^{2}\right\},
$$


which implies that $\left\{\rho_{k}\left(x^{*}, y^{*}\right)\right\}$ is bounded. Hence $\left\{x_{k}\right\},\left\{y_{k}\right\},\left\{u_{k}\right\},\left\{v_{k}\right\},\left\{w_{k}\right\}$ and $\left\{p_{k}\right\}$ are all bounded. By (3.2), (3.4), (3.6), (3.7) and (3.9) we have

$$
\begin{aligned}
& \rho_{\mathrm{k}+1}\left(x^{*}, \mathrm{y}^{*}\right) \leqslant\left\|w_{\mathrm{k}}-x^{*}\right\|^{2}+\left(1-\alpha_{\mathrm{k}}\right)\left(\mu-\alpha_{\mathrm{k}}\right)\left\|\mathrm{U}\left(w_{\mathrm{k}}\right)-w_{\mathrm{k}}\right\|^{2} \\
& +\left\|p_{k}-y^{*}\right\|^{2}+\left(1-\beta_{k}\right)\left(v-\beta_{k}\right)\left\|T\left(p_{k}\right)-p_{k}\right\|^{2} \\
& \leqslant\left(1-t_{k}\right)\left(\left\|u_{k}-x^{*}\right\|^{2}+\left\|v_{k}-y^{*}\right\|^{2}\right)+t_{k}\left(\left\|x^{*}\right\|^{2}+\left\|y^{*}\right\|^{2}\right) \\
& +\left(1-\alpha_{k}\right)\left(\mu-\alpha_{k}\right)\left\|\mathrm{U}\left(w_{k}\right)-w_{k}\right\|^{2}+\left(1-\beta_{k}\right)\left(v-\beta_{k}\right)\left\|\mathrm{T}\left(p_{k}\right)-p_{k}\right\|^{2} \\
& \leqslant\left(1-t_{k}\right) \rho_{k}\left(x^{*}, y^{*}\right)-\left(1-t_{k}\right) \gamma_{k}\left[2-\gamma_{k}\left(\lambda_{A}+\lambda_{B}\right)\right]\left\|A x_{k}-B y_{k}\right\|^{2}+t_{k}\left(\left\|x^{*}\right\|^{2}+\left\|y^{*}\right\|^{2}\right) \\
& +\left(1-\alpha_{k}\right)\left(\mu-\alpha_{k}\right)\left\|\mathrm{U}\left(w_{k}\right)-w_{k}\right\|^{2}+\left(1-\beta_{k}\right)\left(v-\beta_{k}\right)\left\|\mathrm{T}\left(p_{k}\right)-p_{k}\right\|^{2} \text {. }
\end{aligned}
$$

By setting $\Gamma_{k}=\rho_{k}\left(x^{*}, y^{*}\right)$, from (3.10) we have

$$
\begin{aligned}
\left(1-t_{k}\right) \gamma_{k} & {\left[2-\gamma_{k}\left(\lambda_{A}+\lambda_{B}\right)\right]\left\|A x_{k}-B y_{k}\right\|^{2}+\left(1-\alpha_{k}\right)\left(-\mu+\alpha_{k}\right)\left\|U\left(w_{k}\right)-w_{k}\right\|^{2} } \\
& +\left(1-\beta_{k}\right)\left(-v+\beta_{k}\right)\left\|T\left(p_{k}\right)-p_{k}\right\|^{2} \leqslant\left(1-t_{k}\right) \Gamma_{k}-\Gamma_{k+1}+t_{k}\left(\left\|x^{*}\right\|^{2}+\left\|y^{*}\right\|^{2}\right) .
\end{aligned}
$$

Next we will divide the proof into two cases to establish strong convergence.

Case I. Suppose that there exists $n_{0} \in \mathbb{N}$ such that $\Gamma_{k+1} \leqslant \Gamma_{k}$ for all $n \geqslant n_{0}$. In this case, $\lim _{k \rightarrow \infty} \Gamma_{k}$ exists and then $\lim _{k \rightarrow \infty}\left(\Gamma_{k+1}-\Gamma_{k}\right)=0$. Hence from (3.11) with assumptions on $\left\{\alpha_{k}\right\},\left\{\beta_{k}\right\},\left\{t_{k}\right\}$ and $\left\{\gamma_{k}\right\}$ we have

$$
\begin{gathered}
\lim _{k \rightarrow \infty}\left\|\mathrm{U}\left(w_{k}\right)-w_{k}\right\|=\lim _{k \rightarrow \infty}\left\|\mathrm{T}\left(\mathrm{p}_{\mathrm{k}}\right)-\mathrm{p}_{\mathrm{k}}\right\|=0, \\
\lim _{k \rightarrow \infty}\left\|\mathrm{A} x_{k}-\mathrm{B} y_{k}\right\|=0 .
\end{gathered}
$$

From (3.13) we have

$$
\left\|u_{k}-x_{k}\right\|=\gamma_{k}\left\|A^{*}\left(A x_{k}-B y_{k}\right)\right\| \leqslant \gamma_{k} \sqrt{\lambda_{A}}\left\|A x_{k}-B y_{k}\right\| \rightarrow 0 .
$$

Then from (3.12) and (3.14) we obtain

$$
\begin{aligned}
\left\|x_{k+1}-x_{k}\right\| & \leqslant\left\|x_{k+1}-w_{k}\right\|+\left\|w_{k}-u_{k}\right\|+\left\|u_{k}-x_{k}\right\| \\
& \leqslant\left(1-\alpha_{k}\right)\left\|\mathrm{u}\left(w_{k}\right)-w_{k}\right\|+t_{k}\left\|u_{k}\right\|+\left\|u_{k}-x_{k}\right\| \rightarrow 0 .
\end{aligned}
$$

It follows from (3.12) and (3.15) that

$$
\begin{aligned}
\left\|x_{k}-w_{k}\right\| & \leqslant\left\|x_{k+1}-x_{k}\right\|+\left\|x_{k+1}-w_{k}\right\| \\
& \leqslant\left\|x_{k+1}-x_{k}\right\|+\left(1-\alpha_{k}\right)\left\|\mathrm{u}\left(w_{k}\right)-w_{k}\right\| \rightarrow 0 .
\end{aligned}
$$

Similarly, we have

$$
\lim _{k \rightarrow \infty}\left\|v_{k}-y_{k}\right\|=\lim _{k \rightarrow \infty}\left\|y_{k+1}-y_{k}\right\|=\lim _{k \rightarrow \infty}\left\|y_{k}-p_{k}\right\|=0
$$

Since $\left\{x_{k}\right\}$ and $\left\{y_{k}\right\}$ are both bounded, for any $\hat{x} \in \omega_{w}\left(x_{k}\right)$ and $\hat{y} \in \omega_{w}\left(y_{k}\right)$, there exists a subsequence of $\left\{\left(x_{k}, y_{k}\right)\right\}$ (without loss of generality still denoted by $\left.\left\{\left(x_{k}, y_{k}\right)\right\}\right)$, such that $x_{k} \rightarrow \widehat{x}$ and $y_{k} \rightarrow \widehat{y}$. From (3.16) and (3.17) we obtain $w_{k} \rightarrow \widehat{x}$ and $p_{k} \rightarrow \widehat{y}$. Then since $U-I$ and $T-I$ are demiclosed at the origins, and by (3.12) we have $U \widehat{x}=\widehat{x}$ and $T \widehat{y}=\widehat{y}$, i.e., $\widehat{x} \in F(U)$ and $\widehat{y} \in F(T)$. Furthermore $A \widehat{x}-B \widehat{y} \in$ $\omega_{w}\left(A x_{k}-B y_{k}\right)$ and weakly lower semicontinuity of the norm imply

$$
\|A \widehat{x}-B \widehat{y}\| \leqslant \liminf _{k \rightarrow \infty}\left\|A x_{k}-B y_{k}\right\|=0,
$$

hence $(\widehat{x}, \widehat{y}) \in \Omega$. 
Next we prove that $\left\{\left(x_{k}, y_{k}\right)\right\}$ converges strongly to $\left(x^{*}, y^{*}\right)$. By (1.6) we have

$$
\begin{aligned}
\left\|w_{k}-x^{*}\right\|^{2} & =\left\|\left(1-t_{k}\right)\left(u_{k}-x^{*}\right)-t_{k} x^{*}\right\|^{2} \\
& =\left(1-t_{k}\right)^{2}\left\|u_{k}-x^{*}\right\|^{2}+t_{k}^{2}\left\|x^{*}\right\|^{2}-2 t_{k}\left(1-t_{k}\right)\left\langle u_{k}-x^{*}, x^{*}\right\rangle \\
& \leqslant\left(1-t_{k}\right)\left\|u_{k}-x^{*}\right\|^{2}+t_{k}^{2}\left\|x^{*}\right\|^{2}-2 t_{k}\left(1-t_{k}\right)\left\langle u_{k}-x^{*}, x^{*}\right\rangle .
\end{aligned}
$$

Similarly, we have

$$
\left\|p_{k}-y^{*}\right\|^{2} \leqslant\left(1-t_{k}\right)\left\|v_{k}-y^{*}\right\|^{2}+t_{k}^{2}\left\|y^{*}\right\|^{2}-2 t_{k}\left(1-t_{k}\right)\left\langle v_{k}-y^{*}, y^{*}\right\rangle
$$

It follows from (3.3), (3.5), (3.8), (3.18) and (3.19) that

$$
\begin{aligned}
\left\|x_{k+1}-x^{*}\right\|^{2}+\left\|y_{k+1}-y^{*}\right\|^{2} \leqslant & \left\|w_{k}-x^{*}\right\|^{2}+\left\|p_{k}-y^{*}\right\|^{2} \\
\leqslant & \left(1-t_{k}\right)\left(\left\|u_{k}-x^{*}\right\|^{2}+\left\|v_{k}-y^{*}\right\|^{2}\right) \\
& -2 t_{k}\left(1-t_{k}\right)\left(\left\langle u_{k}-x^{*}, x^{*}\right\rangle+\left\langle v_{k}-y^{*}, y^{*}\right\rangle\right)+t_{k}^{2}\left(\left\|x^{*}\right\|^{2}+\left\|y^{*}\right\|^{2}\right) \\
\leqslant & \left(1-t_{k}\right)\left(\left\|x_{k}-x^{*}\right\|^{2}+\left\|y_{k}-y^{*}\right\|^{2}\right)+t_{k}\left[2 ( 1 - t _ { k } ) \left(\left\langle u_{k}-x^{*},-x^{*}\right\rangle\right.\right. \\
& \left.\left.+\left\langle v_{k}-y^{*},-y^{*}\right\rangle\right)+t_{k}\left(\left\|x^{*}\right\|^{2}+\left\|y^{*}\right\|^{2}\right)\right] .
\end{aligned}
$$

Since $\left\{\mathfrak{u}_{k}\right\}$ is bounded, we extract a subsequence $\left\{u_{k_{i}}\right\} \subset\left\{u_{k}\right\}$ that converges weakly to $p$ such that

$$
\limsup _{k \rightarrow \infty}\left\langle u_{k}-x^{*},-x^{*}\right\rangle=\lim _{i \rightarrow \infty}\left\langle u_{k_{i}}-x^{*},-x^{*}\right\rangle=\left\langle p-x^{*},-x^{*}\right\rangle .
$$

By (3.14) we have $p \in \omega_{w}\left(x_{k}\right)$. Similarly, we choose a subsequence $\left\{v_{k_{j}}\right\} \subset\left\{v_{k}\right\}$ that converges weakly to q such that

$$
\limsup _{k \rightarrow \infty}\left\langle v_{k}-y^{*},-y^{*}\right\rangle=\lim _{j \rightarrow \infty}\left\langle v_{k_{j}}-y^{*},-y^{*}\right\rangle=\left\langle q-y^{*},-y^{*}\right\rangle .
$$

By (3.17) we have $q \in \omega_{w}\left(y_{k}\right)$. From the above proof, we have $(p, q) \in \Omega$. Therefore, from the above two equalities and (3.1) we have

$$
\begin{aligned}
\limsup _{k \rightarrow \infty}\left(\left\langle u_{k}-x^{*},-x^{*}\right\rangle+\left\langle v_{k}-y^{*},-y^{*}\right\rangle\right) & \leqslant \limsup _{k \rightarrow \infty}\left\langle u_{k}-x^{*},-x^{*}\right\rangle+\limsup _{k \rightarrow \infty}\left\langle v_{k}-y^{*},-y^{*}\right\rangle \\
& =\lim _{i \rightarrow \infty}\left\langle u_{k_{i}}-x^{*},-x^{*}\right\rangle+\lim _{j \rightarrow \infty}\left\langle v_{k_{j}}-y^{*},-y^{*}\right\rangle \\
& =\left\langle p-x^{*},-x^{*}\right\rangle+\left\langle q-y^{*},-y^{*}\right\rangle \leqslant 0 .
\end{aligned}
$$

Let $\lambda_{k}=t_{k}, \delta_{k}=2\left(1-t_{k}\right)\left(\left\langle u_{k}-x^{*}, x^{*}\right\rangle+\left\langle v_{k}-y^{*}, y^{*}\right\rangle\right)+t_{k}\left(\left\|x^{*}\right\|^{2}+\left\|y^{*}\right\|^{2}\right)$. From Lemma 2.7, (3.20), (3.21) and the assumptions on $\left\{t_{k}\right\}$, we have $\left\{\left(x_{k}, y_{k}\right)\right\}$ converges strongly to $\left(x^{*}, y^{*}\right)$.

Case II. Suppose that there exists a subsequence $\left\{\Gamma_{k_{i}}\right\} \subset\left\{\Gamma_{k}\right\}$ such that $\Gamma_{k_{i}}<\Gamma_{k_{i}+1}$, for all $i \in \mathbb{N}$. In this case, let $\tau: \mathbb{N} \rightarrow \mathbb{N}$ be a mapping for all $n \geqslant n_{0}$ (for some $n_{0}$ large enough) by

$$
\tau(\mathrm{n})=\max \left\{k \leqslant n: \Gamma_{k}<\Gamma_{k+1}\right\} .
$$

Then we have from Lemma 2.9 that $\Gamma_{\tau(n)} \leqslant \Gamma_{\tau(n)+1}$ and clearly, $\{\tau(n)\}$ is a nondecreasing sequence such that $\tau(n) \rightarrow \infty$ as $n \rightarrow \infty$. From (3.11) we have

$$
\begin{aligned}
& \left(1-t_{\tau(n)}\right) \gamma_{\tau(n)}\left[2-\gamma_{\tau(n)}\left(\lambda_{A}+\lambda_{B}\right)\right]\left\|A x_{\tau(n)}-B y_{\tau(n)}\right\|^{2} \\
& +\left(1-\alpha_{\tau(n)}\right)\left(-\mu+\alpha_{\tau(n)}\right)\left\|U\left(w_{\tau(n)}\right)-w_{\tau(n)}\right\|^{2} \\
& +\left(1-\beta_{\tau(n)}\right)\left(-v+\beta_{\tau(n)}\right)\left\|T\left(p_{\tau(n)}\right)-p_{\tau(n)}\right\|^{2} \\
& \quad \leqslant\left(1-t_{\tau(n)}\right) \Gamma_{\tau(n)}-\Gamma_{\tau(n)+1}+t_{\tau(n)}\left(\left\|x^{*}\right\|^{2}+\left\|y^{*}\right\|^{2}\right) \\
& \quad \leqslant t_{\tau(n)}\left(\left\|x^{*}\right\|^{2}+\left\|y^{*}\right\|^{2}\right) \rightarrow 0 .
\end{aligned}
$$


Hence

$$
\lim _{n \rightarrow \infty}\left\|\mathrm{U}\left(w_{\tau(n)}\right)-w_{\tau(n)}\right\|=\lim _{n \rightarrow \infty}\left\|\mathrm{T}\left(\mathrm{p}_{\tau(n)}\right)-p_{\tau(n)}\right\|=\lim _{n \rightarrow \infty}\left\|A x_{\tau(n)}-B y_{\tau(n)}\right\|=0
$$

As in the proof of Case I, for any $\bar{x} \in \omega_{w}\left(x_{\tau(n)}\right)$ and $\bar{y} \in \omega_{w}\left(y_{\tau(n)}\right)$, we have $\bar{x} \in \omega_{w}\left(w_{\tau(n)}\right)$ and $\bar{y} \in \omega_{w}\left(p_{\tau(n)}\right)$. Furthermore, we have $(\bar{x}, \bar{y}) \in \Omega$. Similarly we also have

$$
\limsup _{n \rightarrow \infty}\left(\left\langle u_{\tau(n)}-x^{*},-x^{*}\right\rangle+\left\langle v_{\tau(n)}-y^{*},-y^{*}\right\rangle\right) \leqslant 0 .
$$

Now being similar to the proof of (3.20) and $\Gamma_{\tau(n)} \leqslant \Gamma_{\tau(n)+1}$, we obtain

$$
\begin{aligned}
0 \leqslant & \Gamma_{\tau(n)+1}-\Gamma_{\tau(n)} \\
\leqslant & t_{\tau(n)}\left[2 ( 1 - t _ { \tau ( n ) } ) \left(\left\langle u_{\tau(n)}-x^{*},-x^{*}\right\rangle\right.\right. \\
& \left.\left.+\left\langle v_{\tau(n)}-y^{*},-y^{*}\right\rangle\right)+t_{\tau(n)}\left(\left\|x^{*}\right\|^{2}+\left\|y^{*}\right\|^{2}\right)\right]-t_{\tau(n)} \Gamma_{\tau(n)}
\end{aligned}
$$

which implies that

$$
\Gamma_{\tau(n)} \leqslant 2\left(1-t_{\tau(n)}\right)\left(\left\langle u_{\tau(n)}-x^{*},-x^{*}\right\rangle+\left\langle v_{\tau(n)}-y^{*},-y^{*}\right\rangle\right)+t_{\tau(n)}\left(\left\|x^{*}\right\|^{2}+\left\|y^{*}\right\|^{2}\right) .
$$

Therefore, from $\lim _{n \rightarrow \infty} t_{\tau(n)}=0,(3.22)$ and the above inequality we have

$$
0 \leqslant \liminf _{n \rightarrow \infty} \Gamma_{\tau(n)} \leqslant \limsup _{n \rightarrow \infty} \Gamma_{\tau(n)} \leqslant 0
$$

Then we obtain

$$
\lim _{n \rightarrow \infty} \Gamma_{\tau(n)}=\lim _{n \rightarrow \infty} \Gamma_{\tau(n)+1}=0 .
$$

By using Lemma 2.9, we obtain that

$$
\Gamma_{\mathrm{n}} \leqslant \Gamma_{\tau(\mathrm{n})+1} \rightarrow 0(\mathrm{n} \rightarrow \infty) .
$$

This completes the proof.

Remark 3.3. Theorem 3.2 extends and improves Theorem 2.1 in [18] from the following aspects:

(i) The result from the weak convergence to the strong convergence.

(ii) The mappings $\mathrm{U}$ and $\mathrm{T}$ are extended from quasi-nonexpansive mappings to demicontractive mappings.

\section{Applications}

In this section, we turn our attention to provide some applications in some convex and nonlinear analysis problems.

(a) Split feasibility problem

By taking $\mathrm{U}=\mathrm{P}_{\mathrm{C}}$ and $\mathrm{T}=\mathrm{P}_{\mathrm{Q}}$, we have the following simultaneous iterative algorithm for the SEP (1.3):

$$
\left\{\begin{array}{l}
\forall x_{0} \in H_{1}, y_{0} \in H_{2}, \\
u_{k}=x_{k}-\gamma_{k} A^{*}\left(A x_{k}-B y_{k}\right), \\
w_{k}=\left(1-t_{k}\right) u_{k} \\
x_{k+1}=\alpha_{k} w_{k}+\left(1-\alpha_{k}\right) P_{C}\left(w_{k}\right), \\
v_{k}=y_{k}+\gamma_{k} B^{*}\left(A x_{k}-B y_{k}\right), \\
p_{k}=\left(1-t_{k}\right) v_{k} \\
y_{k+1}=\beta_{k} p_{k}+\left(1-\beta_{k}\right) P_{Q}\left(p_{k}\right) .
\end{array}\right.
$$


Theorem 4.1. Let the mappings $A, B,\left\{\gamma_{k}\right\},\left\{t_{k}\right\},\left\{\alpha_{k}\right\}$ and $\left\{\beta_{k}\right\}$ be the same as in Theorem 3.2. Assume that the solution set $\Omega$ of (1.3) is nonempty. Then the sequence $\left\{\left(x_{k}, y_{k}\right)\right\}$ generalized by (4.1) strongly converges to a solution $\mathrm{P}_{\Omega}\left(\theta_{1}, \theta_{2}\right)$ of (1.3).

(b) Null point problem

Given a maximal monotone operator $\mathrm{M}: \mathrm{H}_{1} \rightarrow 2^{\mathrm{H}_{1}}$, it is well-known that its associated resolvent mapping, $J_{\xi}^{M}:=(I+\xi M)^{-1}$, is firmly nonexpansive, and $x \in M^{-1}(0) \Leftrightarrow 0 \in M(x) \Leftrightarrow x=J_{\xi}^{M}(x)$. By taking $\mathrm{U}=\mathrm{J}_{\xi}^{\mathrm{M}}, \mathrm{T}=\mathrm{J}_{\eta}^{\mathrm{N}}$, where $\mathrm{N}: \mathrm{H}_{2} \rightarrow \mathrm{H}_{2}$ is another monotone operator, the null point problem is to

$$
\text { find } x^{*} \in M^{-1}(0), y^{*} \in N^{-1}(0) \text { such that } A x^{*}=B y^{*} \text {, }
$$

and the algorithm takes the following equivalent form:

$$
\left\{\begin{array}{l}
\forall x_{0} \in H_{1}, y_{0} \in H_{2}, \\
u_{k}=x_{k}-\gamma_{k} A^{*}\left(A x_{k}-B y_{k}\right), \\
w_{k}=\left(1-t_{k}\right) u_{k}, \\
x_{k+1}=\alpha_{k} w_{k}+\left(1-\alpha_{k}\right) J_{\xi}^{M}\left(w_{k}\right), \\
v_{k}=y_{k}+\gamma_{k} B^{*}\left(A x_{k}-B y_{k}\right), \\
p_{k}=\left(1-t_{k}\right) v_{k}, \\
y_{k+1}=\beta_{k} p_{k}+\left(1-\beta_{k}\right) J_{\eta}^{N}\left(p_{k}\right) .
\end{array}\right.
$$

Theorem 4.2. Let the mappings $A, B,\left\{\gamma_{k}\right\},\left\{t_{k}\right\},\left\{\alpha_{k}\right\}$ and $\left\{\beta_{k}\right\}$ be the same as in Theorem 3.2. Assume that $\mathrm{M}: \mathrm{H}_{1} \rightarrow 2^{\mathrm{H}_{1}}$ and $\mathrm{N}: \mathrm{H}_{2} \rightarrow \mathrm{H}_{2}$ are two maximal monotone operators, and the solution set $\Omega$ of (4.2) is nonempty. Then the sequence $\left\{\left(x_{k}, y_{k}\right)\right\}$ generalized by (4.3) strongly converges to a solution $\mathrm{P}_{\Omega}\left(\theta_{1}, \theta_{2}\right)$ of (4.2).

(c) Equilibrium problem

We consider the following problem:

$$
\text { Find } x^{*} \in C, y^{*} \in Q \text { such that } F\left(x^{*}, u\right) \geqslant 0, H\left(y^{*}, v\right) \geqslant 0 \text {, and } A x^{*}=B y^{*}, \forall u \in C, v \in Q \text {, }
$$

with $\mathrm{C}, \mathrm{Q}$ closed convex sets and $\mathrm{F}, \mathrm{H}$ belong in the class of bifunctions $\mathrm{G}$ verifying the following usual conditions:

(A1) $\mathrm{G}(x, x)=0$, for all $x \in \mathrm{D}$;

(A2) $G$ is monotone, that is, $G(x, y)+G(y, x) \leqslant 0$, for all $x, y \in D$;

(A3) for each $x, y, z \in D, \lim \sup _{t \rightarrow 0} G(t z+(1-t) x, y) \leqslant G(x, y)$;

(A4) for each $x \in D$, the function $y \mapsto G(x, y)$ is convex and lower semicontinuous.

It is well-known; see, for example, by Lemma 5 in [8], that the associated resolvent operator

$$
\mathrm{S}_{\lambda}^{\mathrm{G}}=\left\{z \in \mathrm{D}: \mathrm{G}(z, \mathrm{y})+\frac{1}{\lambda}\langle y-z, z-x\rangle, \forall y \in \mathrm{D}\right\},
$$

is firmly nonexpansive and its fixed-points are exactly the equilibria of $G$, that is $G\left(y^{*}, v\right) \geqslant 0, \forall v \in D$.

By taking $U=S_{\mu}^{G}, T=S_{\nu}^{H}$, we have the following iterative algorithm for solving the problem (4.4):

$$
\left\{\begin{array}{l}
\forall x_{0} \in H_{1}, y_{0} \in H_{2}, \\
u_{k}=x_{k}-\gamma_{k} A^{*}\left(A x_{k}-B y_{k}\right), \\
w_{k}=\left(1-t_{k}\right) u_{k} \\
x_{k+1}=\alpha_{k} w_{k}+\left(1-\alpha_{k}\right) S_{\mu}^{G}\left(w_{k}\right), \\
v_{k}=y_{k}+\gamma_{k} B^{*}\left(A x_{k}-B y_{k}\right), \\
p_{k}=\left(1-t_{k}\right) v_{k}, \\
y_{k+1}=\beta_{k} p_{k}+\left(1-\beta_{k}\right) S_{v}^{H}\left(p_{k}\right) .
\end{array}\right.
$$


Theorem 4.3. Let the mappings $\mathrm{A}, \mathrm{B},\left\{\gamma_{\mathrm{k}}\right\},\left\{\mathrm{t}_{\mathrm{k}}\right\},\left\{\alpha_{\mathrm{k}}\right\}$ and $\left\{\beta_{\mathrm{k}}\right\}$ be the same as in Theorem 3.2. Let $\mathrm{C} \subset \mathrm{H}_{1}, \mathrm{Q} \subset \mathrm{H}_{2}$ be two nonempty closed convex sets. Assume that $\mathrm{F}: \mathrm{C} \times \mathrm{C} \rightarrow \mathbb{R}$ and $\mathrm{H}: \mathrm{Q} \times \mathrm{Q} \rightarrow \mathbb{R}$ are two bifunctions satisfying (A1)-(A4), and the solution set $\Omega$ of (4.4) is nonempty. Then the sequence $\left\{\left(x_{k}, y_{k}\right)\right\}$ generalized by (4.5) strongly converges to a solution $\mathrm{P}_{\Omega}\left(\theta_{1}, \theta_{2}\right)$ of (4.4).

Remark 4.4. Since $\mathrm{P}_{\mathrm{C}}$ and $\mathrm{P}_{\mathrm{Q}}$ in Theorem 4.1, $\mathrm{J}_{\xi}^{\mathrm{M}}$ and $\mathrm{J}_{\eta}^{\mathrm{N}}$ in Theorem 4.2, and $\mathrm{S}_{\mu}^{\mathrm{G}}$ and $\mathrm{S}_{v}^{\mathrm{H}}$ in Theorem 4.3 are all firmly nonexpansive, then they are nonexpansive, too. From Lemma 2.10, they are demiclosed at the origins. Thus from Theorem 3.2 we have the results of Theorems 4.1, 4.2 and 4.3.

\section{Acknowledgement}

The authors are extremely grateful to the referees for their useful suggestions that improved the content of the paper. The first author was supported by the Natural Science Foundation of China (no. 11401388, 11671365) and Zhejiang Provincial Natural Science Foundation of China (no. LQ13A010007). The second author was partly supported by a research Grant of Pukyong National University (2016 Year).

\section{References}

[1] C. Byrne, Iterative oblique projection onto convex sets and the split feasibility problem, Inverse Problems, 18 (2002), 441-453. 1, 1

[2] Y. Censor, T. Bortfeld, B. Martin, A. Trofimov, A unified approach for inversion problems in intensity-modulated radiation therapy, Phys. Med. Biol., 51 (2006), 2353-2365. 1

[3] Y. Censor, T. Elfving, A multiprojection algorithm using Bregman projections in a product space, Numer. Algorithms, 8 (1994), 221-239. 1, 1

[4] Y. Censor, A. Segal, The split common fixed point problem for directed operators, J. Convex Anal., 16 (2009), 587-600. 1, 1

[5] K. Goebel, W. A. Kirk, Topics in metric fixed point theory, Cambridge Studies in Advanced Mathematics, Cambridge University Press, Cambridge, (1990). 2.10

[6] P. E. Maingé, Strong convergence of projected subgradient methods for nonsmooth and nonstrictly convex minimization, Set-Valued Anal., 16 (2008), 899-912. 2.9

[7] G. Marino, H.-K. Xu, Weak and strong convergence theorems for strict pseudo-contractions in Hilbert spaces, J. Math. Anal. Appl., 329 (2007), 336-346. 2.2, 2.8

[8] A. Moudafi, Second-order differential proximal methods for equilibrium problems, JIPAM. J. Inequal. Pure Appl. Math., 4 (2003), 7 pages. 4

[9] A. Moudafi, A relaxed alternating CQ-algorithm for convex feasibility problems, Nonlinear Anal., 79 (2013), 117-121. 1

[10] A. Moudafi, Alternating CQ-algorithms for convex feasibility and split fixed-point problems, J. Nonlinear Convex Anal., 15 (2014), 809-818. 1, 1

[11] A. Moudafi, E. Al-Shemas, Simultaneous iterative methods for split equality problem, Trans. Math. Program. Appl., 1 (2013), 1-11. 1

[12] Y. Shehu, F. U. Ogbuisi, An iterative algorithm for approximating a solution of split common fixed point problem for demi-contractive maps, Dyn. Contin. Discrete Impuls. Syst. Ser. B Appl. Algorithms, 23 (2016), 205-216. 1

[13] W. Takahashi, Nonlinear functional analysis, Yokohama Publishers, Yokohama, Japan, (2000). 2

[14] H.-K. Xu, Iterative algorithms for nonlinear operators, J. London Math. Soc., 66 (2002), 240-256. 2.7

[15] Y.-H. Yao, R. P. Agarwal, M. Postolache, Y.-C. Liou, Algorithms with strong convergence for the split common solution of the feasibility problem and fixed point problem, Fixed Point Theory Appl., 2014 (2014), 14 pages. 1

[16] Y.-H. Yao, M. Postolache, Y.-C. Liou, Strong convergence of a self-adaptive method for the split feasibility problem, Fixed Point Theory Appl., 2013 (2013), 12 pages.

[17] Y.-H. Yao, J. Wu, Y.-C. Liou, Regularized methods for the split feasibility problem, Abstr. Appl. Anal., 2012 (2012), 13 pages. 1

[18] J. Zhao, S.-N. He, Simultaneous iterative algorithms for the split common fixed-point problem governed by quasinonexpansive mappings, J. Nonlinear Convex Anal., (accepted). 1, 3.3 